\title{
Concatenated retrieval of correlated stored information in neural networks
}

\author{
R. M. C. de Almeida, ${ }^{1, *}$ A. Espinosa, ${ }^{2}$ and M. A. P. $\operatorname{Idiart}^{1}$ \\ ${ }^{1}$ Instituto de Física, Universidade Federal do Rio Grande do Sul, Caixa Postal 15051, 91501-970 Porto Alegre, RS, Brazil \\ ${ }^{2}$ Instituto de Física, Universidade Federal da Bahia, 40210-340 Salvador, BA, Brazil
}

(Received 20 March 2006; revised manuscript received 4 September 2006; published 19 October 2006)

\begin{abstract}
We consider a coupled map lattice defined on a hypercube in $M$ dimensions, taken here as the information space, to model memory retrieval and information association by a neural network. We assume that both neuronal activity and spike timing may carry information. In this model the state of the network at a given time $t$ is completely determined by the intensity $y(\sigma, t)$ with which the information pattern represented by the integer $\sigma$ is being expressed by the network. Logistic maps, coupled in the information space, are used to describe the evolution of the intensity function $y(\vec{\sigma}, t)$ with the intent to model memory retrieval in neural systems. We calculate the phase diagram of the system regarding the model ability to work as an associative memory. We show that this model is capable of retrieving simultaneously a correlated set of memories, after a relatively long transient that may be associated to the retrieving of concatenated memorized patterns that lead to a final attractor.
\end{abstract}

DOI: 10.1103/PhysRevE.74.041912

PACS number(s): 87.18.Sn, 05.45.Ra

\section{INTRODUCTION}

In a previous paper [1] we have proposed that to any information processing machine we can associate a space where each point represents an information pattern that the machine may express. This information space may be viewed as an analogy to the Fourier space for a musical instrument. The advantage of such approach is to provide a framework to compare the performance of different information processors, realized through different physical substrates such as, for example, neural networks or the immune system, exactly in the same way as the Fourier space allows the comparison of music played in a violin or a flute.

The topology defining neighborhood relations between information states as well as the interactions between these neighbors may strongly vary from machine to machine. Understanding, from one side, what interactions in the information space allow the systems to accomplish different tasks and, from the other side, discovering the role played in the information space by the physical, chemical, or biological interactions of the material system, is a first step not only to understand the functioning of an existing system, but also to build an information processing machine with designed capabilities.

An important step to this understanding is to write the recipe to go from the material configuration space to the information space, that is, the analogous to the Fourier transform in the metaphor of musical instruments. This step should be taken with care, paying due attention to the biological facts in the special case of neural networks or other biological systems. This will prove to be an enormous task and in Ref. [1] and here we just propose a starting point. A second step is to understand the dynamics in the information space.

In Ref. [1] we have proposed the topology of an $M$-dimensional hypercube to model the information space of

\footnotetext{
*Electronic address: rita@if.ufrgs.br
}

a system capable of expressing a maximum of $2^{M}$ information patterns. Each vertex of the hypercube is associated to the integer $\sigma$ whose binary representation is the $M$-dimensional vector giving the position of that vertex, while the neighbors of a given $\sigma$ are the $M$ integers whose binary representation differ from $\sigma$ by one bit. To each point $\sigma$ in this information space, we associate a continuous, non negative function representing the intensity of expression $y(\sigma, t)$ of that information pattern at a given time $t$. Consequently we look for a transformation (and its inverse) that takes from the $2^{M}$ values of the intensity of expression to the variables that describe the system in the real world (and vice versa).

As the vertices of a hypercube in $M$ dimensions are labeled by bit strings it is all too tempting to associate each vertex to the configuration of a network made of $M$ binary neurons, obtaining a trivial transformation from the configuration space to the information space. In fact this is what is implicitly done in Hopfield-like models [2-5]. Observe, however, that when this simple transformation is assumed, (i) there is no room to expressing more than one information pattern at a given time $[y(\sigma, t)$ is different from zero for only one $\sigma]$, and (ii) there are $M$ variables in the configuration space, while we find $2^{M}$ intensity of expression functions. In fact, the smaller number of variables in the configuration space reduces the number of states in the information space available to the system, strongly impoverishing its computation performance. On the other hand, the ability of simultaneously expressing different information patterns is a necessary condition to build up a representation of the environment that is isomorphic to the real world in some degree. These facts point to the necessity of information processing units in the material world far more complex than binary neurons.

Fortunately, although neurons do behave as binary variables during time intervals of the order of $2 \mathrm{~ms}$, real neural networks dynamics is rather more complex than that of Hopfield-like models. In fact there is evidence that neurons integrate signals in space and time with under millisecond 
precision in spiking synchronization [6-12]. In what regards space integration, Mountcastle [13-15] had already proposed minicolumns as sets of neurons more intensely coupled between themselves than with other neurons and many authors have found evidences for these spatially integrated structures beyond the sensory cortex [16-21]. Furthermore, developments in neuroscience reveal that it is important to consider multi-interactions and a dynamical modulation of these interactions, in the sense that it is the overall activity of the network that should define how these interactions are regulated [22-33]. It makes sense, then, the definition of sets of neurons acting coherently as what we call cognitive units. Now the possible states for a system made of such cognitive units compose the configuration space, from which it is possible to define a transformation that takes to an information space where different patterns may be simultaneously expressed.

In Ref. [1] we have approached the problem from the information space side, by proposing a logistic map model for the dynamics of the expression intensity function $y(\sigma, t)$, where memorized information patterns present higher logistic parameters than nonmemorized patterns. We also proposed a transformation to the configuration space, where the role played by multi-interactions, space and time integrations, and synaptic modulation clearly emerge, showing why a Hopfield-like dynamics is not suitable and more complex cognitive units are required. Nevertheless, in the adequate limits we recover a Hopfield like model, in the narrow sense that there are attractors of the model dynamics which are patterns stored through a Hebb-like learning rule, including multi-interaction terms. Finally we compare the results with experimental data regarding the performance of human subjects in remembering the items of a list that has been previously shown. This is the first neural network model, to our knowledge, that allows such comparisons.

In this paper we consider a slightly different dynamics, that have turned out to present a better performance regarding associative memory capabilities. For this dynamics, in Sec. II we propose the model equations and in Sec. III we obtain a phase diagram in the model parameter space. Section IV presents results regarding the effect of loading the network with memorized patterns and of external noise, and finally, in Secs. V and VI we calculate the size of basins of attractions and discuss effective size of basins of attraction when the network is capable of concatenating a chain of successively retrieved correlated memories. Discussions and conclusions are presented in Sec. VII.

\section{THE TRANSFORMATION AND THE MODEL EQUATIONS}

Space and time integration of the signals received by a neuron greatly influences its response. As we have extensively discussed in Ref. [1], we can define a cognitive unit that contemplates the states of $\nu$ binary neurons during $K$ slices of a time interval of typically $15 \mathrm{~ms}$. The state of the cognitive unit $\tilde{S}_{i}(t)$ during a time step beginning at time $t$ is then defined by a string of $K \times \nu$ bits, written as

$$
\begin{aligned}
\tilde{S}_{i}(t)= & \left(s_{1,1}^{i}, s_{1,2}^{i}, \ldots,\right. \\
& \left.s_{1, K}^{i}, s_{2,1}^{i}, s_{2,2}^{i}, \ldots, s_{2, K}^{i}, \ldots, s_{\nu, 1}^{i}, s_{\nu, 2}^{i}, \ldots, s_{\nu, K}^{i}\right),
\end{aligned}
$$

where $s_{j, \tau}^{i}= \pm 1$ indicates whether the $j$ th neuron in processing unit $i$ has spiked in the $\pi$ th time subinterval.

We assume as in Ref. [1] that these cognitive units are the relevant units to process information and it is through them that we have proposed the transformation to the information space, defined as the vertices of a hypercube in $M$ dimensions. Each vertex $\sigma, \sigma=0,1,2, \ldots, 2^{M}-1$, is associated to an information pattern that can be expressed by the neural network and a continuous intensity function is defined for each pattern as $y(\sigma, t)$. The transformation is written as

$$
\begin{gathered}
a(t)=\sum_{\sigma=0}^{2^{M}-1} y(\sigma, t), \\
\left\langle\widetilde{S}_{i}(t)\right\rangle a(t)=\sum_{\sigma=0}^{2^{M}-1} y(\sigma, t) \sigma_{i}, \\
\left\langle\widetilde{S}_{i}(t) \widetilde{S}_{j}(t)\right\rangle a(t)=\sum_{\sigma=0}^{2^{M}-1} y(\sigma, t) \sigma_{i} \sigma_{j}, \\
\vdots \\
\left\langle\widetilde{S}_{1}(t) \tilde{S}_{2}(t) \cdots \tilde{S}_{M}(t)\right\rangle a(t)=\sum_{\sigma=0}^{2^{M}-1} y(\sigma, t) \sigma_{1} \sigma_{2} \cdots \sigma_{M},
\end{gathered}
$$

where $\sigma_{i}= \pm 1$ for $i=1, \ldots, M$ give the binary representation of $\sigma$ through the relation

$$
\sigma=\sum_{i=1}^{M} \frac{\sigma_{i}+1}{2} 2^{i-1}
$$

and the correlation functions are defined as

$$
\left\langle\widetilde{S}_{i_{1}}(t) \widetilde{S}_{i_{2}}(t) \cdots \widetilde{S}_{i_{m}}(t)\right\rangle=\frac{1}{\nu K} \sum_{j=1}^{\nu} \sum_{\tau=1}^{K} s_{j, \tau}^{i_{1}} j_{j, \tau}^{i_{2}} \cdots s_{j, \tau}^{i_{m}},
$$

where $i_{1}, i_{2}, \ldots, i_{m}$ correspond to different processing units. As each bit $s_{j, \tau}^{i}= \pm 1$, the above equation implies that correlation functions with one or more repeated units are redundant. For a network with $N$ neurons, there are $M=N / \nu$ processing units, and $M$ correlation functions involving only one unit, but a total number of $2^{M}$ nonredundant correlation functions. This is so because the correlation functions that involve more than one unit carry information on the spiking relative phases.

The number of correlation functions in the left hand side of Eqs. (2) must be equal to the number of averages in the information space that lays in the right hand side, what explains why we took the bit-string length $M$ in the information space equal to the number $N / \nu$ of processing units, where $N$ is the total number of neurons in the network.

The role played by $K$, the number of time slices in the integration time, is to approach the correlation functions to the continuous limit $(K \nu \rightarrow \infty)$. Observe that, given all experimental quantities, in the continuous limit, we can univocally determine $y(\sigma, t)$ up to a normalization constant $a(t)$. 
On the other hand, $a(t)$ can be viewed as an overall information activity of the network, as measured in the information space. Equations (2) also show that the intensity of expression functions $y(\sigma, t)$ are analogs of the oscillation modes amplitudes in a Fourier transform, meaning that they completely determine the variables in the configuration space and vice versa.

There is a strong degree of arbitrariness in choosing Eqs. (2) and (4). They imply, for example, a one to one correspondence between neurons in different cognitive units. In the general case, a different set of correlation functions would be necessary to characterize the network behavior, yielding perhaps modified transformation equations, in which case $M$ $\neq N / \nu$. In fact, the information space dimension, $M$, should be equal to the logarithm of the number of independent variables of the evolution equations describing the neural network.

The dynamics for the intensity functions should meet the following, in the case we design the network as an associative memory device.

(i) There must be differences between memorized and non-memorized patterns such that attractors correspond to the expression of memorized patterns. The retrieving of a pattern $\sigma$ is modeled by a non-negligible value of the intensity function $y(\sigma, t)$ for a finite time interval. The dynamics of the model must present attractors associated to the retrieving of memorized patterns.

(ii) There must be a range of external noise where memory retrieving attractors are robust.

(iii) There must be a range of external noise where nonmemorized patterns are not excited, preventing an activity explosion.

(iv) The excitation of neighbors should temporarily elicit information patterns, memorized or not, to allow information transport along the hypercube, and the consequent memory retrieval by association.

Here we investigate the properties of the information space whose intensity function dynamics is described by a set of $2^{M}$ logistic maps coupled by the logistic parameter as follows:

$$
y(\sigma, t+1)=[1-y(\sigma, t)] y(\sigma, t) \lambda(\sigma, t),
$$

where

$$
\lambda(\sigma, t)=x(\sigma)+\frac{\sum_{\sigma^{\prime}} z\left(\sigma, \sigma^{\prime}\right) y\left(\sigma^{\prime}, t\right)}{\sum_{\sigma^{\prime}} y\left(\sigma^{\prime}, t\right)} .
$$

Depending on whether $\lambda(\sigma, t)$ is momentarily less, equal or greater than 1 , there may be persistent states where $y(\sigma, t)$ is larger than zero. The logistic parameter is the result of the combination of two terms: (i) $x(\sigma)$, that does not depend on time and is a function of $\sigma$ only and (ii) a dynamically set term, that describes the coupling between different information patterns. Here we assume a number $P$ of special patterns that the system is more inclined to express, the memorized patterns, represented by $\sigma^{\mu}$ with $\mu=1,2, \ldots, P$. As in Ref. [1] such special status is accomplished by setting

$$
x(\sigma)=k_{v}+\left(k_{m}-k_{v}\right) \sum_{\mu=1}^{P} \delta\left(\sigma, \sigma^{\mu}\right),
$$

where $\delta\left(\sigma, \sigma^{\mu}\right)$ is the Kronecker delta function, while $k_{m}$ and $k_{v}$ are adjustable parameters of the model. The second term describes how the state of the whole network influences the effective retrieving of a given pattern. Here we consider that such interaction is only possible between neighboring states, such that patterns with similar information content reinforce each other, therefore

$$
z\left(\sigma, \sigma^{\prime}\right)=z \delta\left(\sigma^{\prime}, \sigma^{(i)}\right),
$$

where $z$ is a positive parameter and the integers $\sigma^{(i)}=\sigma$ $-\sigma_{i} 2^{i-1}, i=1, \ldots, M$ are the first neighbors of $\sigma$ in the hypercube.

A striking difference between the information space as conceived in this work and a phase space for usual neural network models, is that a given state of the physical system realizing the information processing may simultaneously express multiple information patterns. On the other hand, there must be a limit for that and therefore some competition between information patterns is essential. This is achieved by using the network information activity $a(t)=\Sigma_{\sigma=1}^{2^{M}} y(\sigma, t)$ to modulate the coupling between neighboring sites in the information space: when many different patterns are simultaneously expressed, the network is too active and association is less intense. Equation (5) differs from Ref. [1] in the saturation term (the first factor in the right-hand side): $[1-a(t)]$ was replaced by $[1-y(\sigma, t)]$. In the original model the saturation term added a competition among patterns. This change enhances information transport between different memory patterns as we will see in the results towards the end of this paper.

The correlation functions are relevant in determining the evolution of the system. In fact, for the evolution equations given in Eq. (5) to completely specify $y(\sigma, t+1)$ (up to the normalization constant), it is necessary to know all pattern intensities at time $t$. As there are $2^{M}$ intensities, the complete specification of the state of the net requires a phase space of $2^{M}$ dimensions, where Eq. (5) can be regarded as a master equation of a Markov process. Observe that the set of the quantities $a(t),\left\langle\widetilde{S}_{i}\right\rangle_{t},\left\langle\widetilde{S}_{i} \widetilde{S}_{j}\right\rangle_{t},\left\langle\widetilde{S}_{i} \widetilde{S}_{j} \widetilde{S}_{k}\right\rangle_{t}, \ldots,\left\langle\widetilde{S}_{1} \tilde{S}_{2} \cdots \widetilde{S}_{M}\right\rangle_{t}$ contains $2^{M}$ elements and allows us to determine the $2^{M}$ values of $y(\sigma, t)$ at a given time $t$ and their subsequent evolution.

That the evolution equation, Eq. (5), defined in the information space, implies an evolution for the cognitive units variables, can be seen by first writing the $\delta$ function in Eq. (7) as

$$
\begin{aligned}
\delta\left(\sigma, \sigma^{\mu}\right)= & \prod_{i=1}^{M} \frac{1+\sigma_{i} \sigma_{i}^{\mu}}{2} \\
= & \frac{1}{2^{M}}\left[1+\sum_{i=1}^{M} \sigma_{i} \sigma_{i}^{\mu}+\sum_{i=1}^{M-1} \sum_{j=i+1}^{M} \sigma_{i} \sigma_{i}^{\mu} \sigma_{j} \sigma_{j}^{\mu}\right. \\
& \left.+\cdots+\sigma_{1} \sigma_{1}^{\mu} \sigma_{2} \sigma_{2}^{\mu} \cdots \sigma_{M} \sigma_{M}^{\mu}\right]
\end{aligned}
$$


such that using this expansion in Eq. (7), we may rewrite $x(\sigma)$ as

$$
\begin{aligned}
x(\sigma)= & k_{v}+\frac{2 P}{2^{M}}\left(k_{m}-k_{v}\right)\left[1+\sum_{i=1}^{M} J_{i}^{(1)} \sigma_{i}+\sum_{i=1}^{M-1} \sum_{j=i+1}^{M} J_{i j}^{(2)} \sigma_{i} \sigma_{j}\right. \\
& +\sum_{i=1}^{M-2} \sum_{j=i+1}^{M-1} \sum_{k=j+1}^{M} J_{i j k}^{(3)} \sigma_{i} \sigma_{j} \sigma_{k} \\
& \left.+\cdots+J_{12 \cdots M}^{(M)} \sigma_{1} \sigma_{2} \cdots \sigma_{M}\right]
\end{aligned}
$$

where the synaptic intensities $J^{(k)}$ describe multiinteractions involving $k$ neurons and are given as

$$
J_{j}^{(1)}=\frac{1}{P} \sum_{\mu=1}^{P} \sigma_{i}^{\mu},
$$

$$
\begin{gathered}
J_{i j}^{(2)}=\frac{1}{P} \sum_{\mu=1}^{P} \sigma_{i}^{\mu} \sigma_{j}^{\mu}, \\
J_{i j k}^{(3)}=\frac{1}{P} \sum_{\mu=1}^{P} \sigma_{i}^{\mu} \sigma_{j}^{\mu} \sigma_{k}^{\mu}, \\
\vdots \\
J_{12 \cdots M}^{(M)}=\frac{1}{P} \sum_{\mu=1}^{P} \sigma_{1}^{\mu} \sigma_{2}^{\mu} \cdots \sigma_{M}^{\mu},
\end{gathered}
$$

which are expressions equivalent to the ones presented in Refs. [27-33].

The expansion for $x(\sigma)$ proposed in Eq. (10) implies that the first term in the evolution equation (5) is regulated by Hebb-like terms. Using Eq. (10) in Eq. (5) we obtain

$$
\begin{aligned}
a(t+1)\left\langle\tilde{S}_{i}\right\rangle_{t+1}= & {\left[k_{v}+\frac{2 P}{2^{M}}\left(k_{m}-k_{v}\right)\right] a(t)\left\langle\tilde{S}_{i}\right\rangle_{t}+\frac{2 P}{2^{M}}\left(k_{m}-k_{v}\right) a(t)\left[\sum_{j=1}^{M} J_{j}^{(1)}\left\langle\tilde{S}_{i} \tilde{S}_{j}\right\rangle_{t}+\sum_{j=1}^{M-1} \sum_{k=j+1}^{M} J_{j k}^{(2)}\left\langle\tilde{S}_{i} \tilde{S}_{j} \tilde{S}_{k}\right\rangle_{t}+\sum_{j=1}^{M-2} \sum_{k=j+1}^{M-1} \sum_{l=k+1}^{M} J_{j k l}^{(3)}\right.} \\
& \left.\times\left\langle\widetilde{S}_{i} \widetilde{S}_{j} \widetilde{S}_{k} \tilde{S}_{l}\right\rangle_{t}+\cdots\right]+\frac{2 P}{2^{M}}\left(k_{m}-k_{v}\right) \sum_{\sigma=0}^{2^{M}-1} y^{2}(\sigma, t)\left[\sum_{j=1}^{M} J_{j}^{(1)} \sigma_{j} \sigma_{i}+\sum_{j=1}^{M-1} \sum_{k=j+1}^{M} J_{j k}^{(2)} \sigma_{j} \sigma_{k} \sigma_{i}+\sum_{j=1}^{M-2} \sum_{k=j+1}^{M-1} \sum_{l=k+1}^{M} J_{j k l}^{(3)} \sigma_{j} \sigma_{k} \sigma_{l} \sigma_{i}\right. \\
& +\cdots]+\sum_{\sigma=0}^{2^{M}-1}[1-y(\sigma, t)] y(\sigma, t) \frac{z \sigma_{i}}{a(t)} \sum_{j}^{M} y\left(\sigma^{(j)}, t\right)
\end{aligned}
$$

Neglecting terms of order 2 or higher in $y(\sigma, t)$, the above equation shows that the evolution of the correlation function $\left\langle\tilde{S}_{i}\right\rangle_{t}$ is regulated by the value of correlations in a previous time step. Multi-interaction is clearly present and also the interaction modulation between any two given cognitive units by the state of other units. In this paper we will proceed by analyzing the performance of the model in the information space and in the next section we define useful order parameters.

\section{THE PHASE DIAGRAM IN THE ABSENCE OF NOISE}

Depending on the parameters of the model, many different attractors may appear. Here we shall focus on those attractors and their stability that are related to the performance of the model as an associative memory device. Given the nature of the evolution equations, which are coupled logistic maps, we can expect a trivial attractor where all the expression intensity functions are zero and no information is expressed by the net. This inactive state should be stable against an explosion of information expression, where intensity functions of all nonmemorized patterns have nonneglegible values. For that, the state where $y(\sigma, t)=y^{*}$ with $y^{*}>0$ if $\sigma \neq \sigma^{\mu}, \mu=1,2, \ldots, P$ should not be an attractor of the dynamics. For this requirement it suffices that $k_{v}<1$, in the limit that $z M / 2^{M} \rightarrow 0$, that is, if $M$ is large enough.

We consider first a low memory load: $P$ is small enough such that $P$ randomly chosen memorized patterns are not near one another on the hypercube. In this case we shall consider states where one memorized pattern, say $\sigma^{1}$, is expressed with intensity $y_{0}$ while its $M$ first neighbors are expressed with intensity $y_{1}$. All other patterns are not expressed by the net, such that the global activity $a(t)=y_{0}+M y_{1}$. The evolution equations are then reduced to

$$
\begin{aligned}
& y_{0}=y_{0}\left(1-y_{0}\right)\left[k_{m}+z \frac{M y_{1}}{y_{0}+M y_{1}}\right], \\
& y_{1}=y_{1}\left(1-y_{1}\right)\left[k_{v}+z \frac{y_{0}}{y_{0}+M y_{1}}\right],
\end{aligned}
$$

with the condition that $k_{v}+z \frac{2 y_{1}}{y_{0}+M y_{1}}<1$ ensuring that the intensity of expressions of second neighbors to $\sigma^{1}$ goes to zero $\left(y_{2}=0\right)$. Consider first the solution where $y_{0} \neq 0$ but $y_{1} \rightarrow 0$. For this solution to exist we need $k_{m}>1$ and $k_{v}+z<1$, yielding $y_{0}=\left(k_{m}-1\right) / k_{m}$. When $y_{1}>0$, we must explicitly solve Eq. (12). Isolating $y_{0}$ in the second equation of Eq. (12) and using in the first equation we obtain an algebraic third order 
equation for $M y_{1}$. Observe that $y_{1} \rightarrow 0$ when $M \rightarrow \infty$, but it is possible that $M y_{1}$ remains finite.

$$
\left(M y_{1}\right)^{3}+A_{2}\left(M y_{1}\right)^{2}+A_{1}\left(M y_{1}\right)+A_{0}=0,
$$

where the coefficients are

$$
\begin{gathered}
A_{2}=\left(\frac{1-2 k_{v}}{k_{v}}+\frac{1}{k_{m}+k_{v}+z}\right) M+\frac{k_{v}+z-1}{k_{v}}+\frac{k_{m}}{k_{v}\left(k_{m}+k_{v}+z\right)}, \\
A_{1}=\frac{\left(1-k_{v}\right)\left(1-k_{m}-k_{v}-z\right)}{k_{v}\left(k_{m}+k_{v}+z\right)} M^{2}+\left(\frac{2\left(2-k_{v}-z\right)}{k_{v}}\right. \\
\left.-\frac{3 k_{m}+1}{\left(k_{m}+k_{v}+z\right) k_{v}}\right) M \\
A_{0}=\left(\frac{k_{v}+z-3}{k_{v}}+\frac{2\left(k_{m}+1\right)}{\left(k_{m}+k_{v}+z\right) k_{v}}\right) M^{2} .
\end{gathered}
$$

In the $M \rightarrow \infty$ limit Eq. (13) reads

$$
\begin{aligned}
& \frac{\left(1-k_{v}\right)\left(1-k_{m}-k_{v}-z\right)}{k_{v}\left(k_{m}+k_{v}+z\right)} M y_{1}+\left(\frac{k_{v}+z-3}{k_{v}}+\frac{2\left(k_{m}+1\right)}{\left(k_{m}+k_{v}+z\right) k_{v}}\right) \\
& \quad=0,
\end{aligned}
$$

yielding the solution

$$
\begin{aligned}
& y_{0}=\frac{k_{m}+k_{v}+z-2}{k_{m}+k_{v}+z-1}, \\
& y_{1}=\frac{1}{M} \frac{k_{v}+z-1}{1-k_{v}} y_{0} .
\end{aligned}
$$

Clearly, positive solutions can only exist for $k_{m}+k_{v}+z>2$ and $k_{v}+z>1$, since $k_{v}<1$ to avoid undesirable high excitability of non memorized patterns. We can define an effective logistic map parameter as $\lambda=k_{m}+z M y_{1} /\left(y_{0}+M y_{1}\right)$ that, in order to guarantee stationary solutions, should be in the interval $(0,3)$, yielding the condition $2<k_{m}+k_{v}+z<4$. Equations (16) are valid for $M \rightarrow \infty$. We point, however, that stationarity may represent a too stringent requirement, since periodic or chaotic deviations around a non-negligible value of the intensity function could also be interpreted as pattern retrieval. In this case, one should expect $2<k_{m}+k_{v}+z<5$. Anyway, we shall proceed the calculation of the phase space within the stationarity requirement. In the following sections, where we consider information transport, this limit will be relaxed.

For finite $M$, we must solve Eq. (13), requiring further that sites that are second neighbors to the excited memory also go to zero. The phase diagram for $k_{v}=0.25$ and 0.75 are shown in Fig. 1 for $M=10,16,50$, and $M \rightarrow \infty$ curve. Finite size effects are clearly visible, but the solutions approach the infinite size result very fast. The finite size deviations, arise from nonzero values of $y_{1}$ which can excite second neighbors for high values of $k_{v}$ or $z$ : when $M \rightarrow \infty, y_{1} \rightarrow 0$ and these excitations are not possible.

The implicit summary in the phase diagrams is as follows. There are two different retrieving situations: (i) when $k_{v}+z$ $<1$ and $1<k_{m}<3$, we have the trivial stationary solution

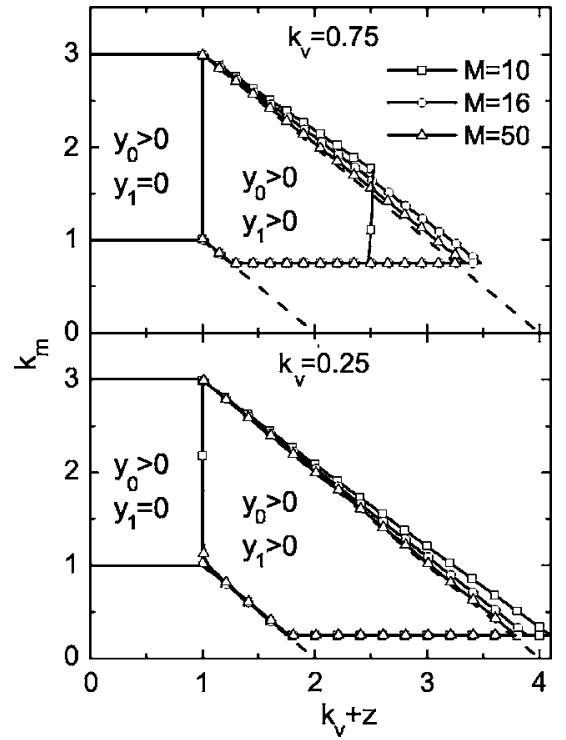

FIG. 1. Phase diagrams for $k_{v}=0.75$ and $k_{v}=0.25$ in the $\left(k_{m}, k_{v}+z\right)$ plane. Lines and open symbols correspond to simulations with different network sizes. The horizontal line in the region $k_{v}+z>1$ corresponds to the line $k_{m}=k_{v}$. Dashed lines corresponds to $k_{m}+k_{v}+z=2$ and $k_{m}+k_{v}+z=4$. For $k_{m}<1$ and $k_{m}+k_{v}+z<2$ there is only the trivial solution, while the upper region $k_{m}+k_{v}+z<4$ corresponds to either trivial or nonstationary solutions.

where $y_{0}=\left(k_{m}-1\right) / k_{m}$ and $y_{1}=0$ and (ii) when $k_{v}+z>1$ and $2-k_{v}-z<k_{m}<4-k_{v}-z$. For the last cases the solutions for $M \rightarrow \infty$ are given by Eq. (16). The horizontal line for $k_{v}+z$ $>1$ in both plots is a lower bound guaranteeing that $k_{m}$ $>k_{v}$, that is, memorized patterns are more excitable than nonmemorized ones.

Finite size effects are better detected in the plot of the solutions themselves, presented in Fig. 2. We have plotted $y_{0}$ and $y_{1}$ for different values of $M$, obtained from either $M$ $\rightarrow \infty$ solution, Eq. (16), or obtained from Eq. (13) for finite $M$. To verify the validity of these solutions we have simulated the model for $M=10$ and 16 , shown as solid symbols in

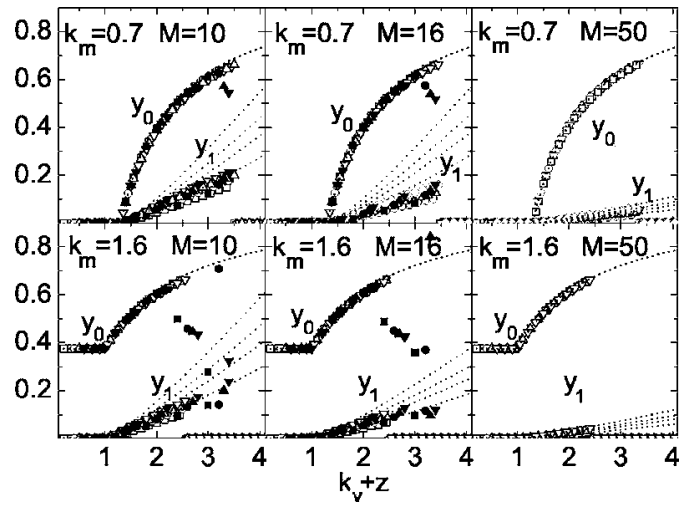

FIG. 2. Solutions $y_{0}$ and $y_{1}$ : Simulation results for finite $M$ $=10$ and 16 (solid symbols); theoretical solutions for $M=10, M$ $=16$, and $M=50$ (open symbols); theoretical solution in the $M$ $\rightarrow \infty$ limit (dashed lines). We considered $k_{v}=0.2$ (squares), 0.4 (circles), 0.5 (up triangles), and 0.6 (down triangles). Larger $k_{v}$ yields larger $y_{1}$. 


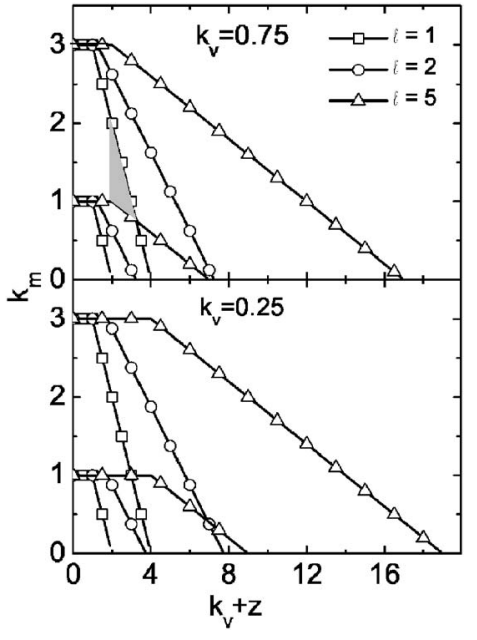

FIG. 3. Phase diagrams for $k_{v}=0.75$ and $k_{v}=0.25$ in the $\left(k_{m}, k_{v}+z\right)$ plane for solutions presenting $l$ excited, isolated memories.

Fig. 2. We considered $k_{v}=0.2,0.4,0.5$, and 0.6. The simulations were performed by iterating Eqs. (5) for long time from initial conditions equal to the $M \rightarrow \infty$ analytical solution and guaranteeing that a stationary state has been attained. We have considered only one memorized pattern, since in this section we focus on the sparsely loaded (small $P$ ) case. We observe that the $M \rightarrow \infty$ values for $y_{0}$ are a very good approximation even for small lattices, while the values for $y_{1}$ are more sensitive to the finite size of the lattice. Differences in $y_{1}$ between finite $M$ and theoretical, $M \rightarrow \infty$ solutions, represented by dashed lines, are larger for larger values of $k_{v}$. In fact, for finite $M$, both theory and simulations show smaller differences between the values of $y_{1}$ obtained with different $k_{v}$ when compared to infinite lattices results.

Now we consider the solution where $l$ noncorrelated patterns are equally and simultaneously expressed by the net. We still restrict ourselves to the sparsely loaded net. In this case the stationary solutions and the phase diagrams are trivially obtained from Eqs. (16) by changing $z \rightarrow z / l$, since the difference arises through the global network activity $a(t)$ and not by the action of the excited memories neighborhood.

Focusing in the $M \rightarrow \infty$ case, we plot in Fig. 3 the phase diagram for different values of $l$, for two different values of $k_{v}$. The point here is to indicate the region in the phase diagram that a system may present stationary solutions with different numbers of simultaneously expressed memories: the stationary solution regions in different $l$ phase diagrams should overlap. It is possible to see in Fig. 3 that for $k_{v}$ $=0.25$ there is an interval for $k_{m}$ and $z$ such that stationary solutions with $l=1$ and 2 are stable, but not $l=5$. Remember, we look for solutions where $y_{0}>0$ and $y_{1}>0$, see Fig. 1 . When $k_{v}=0.75$, on the other hand, there is a small region in the phase diagram, painted in light gray in the figure, where solutions with $l=5$ simultaneously expressed memories are also stable. We can estimate the maximum possible number of simultaneously expressed memories $l_{\max }$ by noting that overlapping regions in the phase space appear when the higher limit for $l=1$ stable solutions are larger than the lower limit for $l>1$ stable solutions. This happens if $l-(l-1) k_{v}$ $<3$, yielding

$$
l_{\max }=\frac{3-k_{v}}{1-k_{v}} .
$$

This limit is rather interesting. Results of experiments where a list containing many items is shown to individuals, in a controlled environment and under well defined conditions, show that after a time interval individuals may remember about five items of the list [34-36]. The above results show that $k_{v} \sim 0.5$ for $l_{\max }=5$, which falls in a reasonable region of the phase space, in what regards the performance of the model.

\section{THE EFFECTS OF LOADING THE NETWORK AND EXTERNAL NOISE}

We shall now consider the effect of loading the network with memorized patterns. For that we repeat the iteration procedure of last section, starting from the state corresponding to the stationary solutions where one memory is expressed with intensity $y_{0}$, surrounded by neighbors expressed with intensity $y_{1}$. The difference here is that we consider that there are $P$ memorized patterns, that is, $P$ vertices of the hypercube have been randomly chosen to have $x(\sigma)=k_{m}$. We then let the system stabilize to measure diverse quantities. In the absence of external noise and for small values of $P$ we do not see any differences in the model behavior. However, when $P$ increases it may happen that memorized patterns are neighbors to each other in the hypercube and hence the assumptions used to obtain the phase diagrams in Figs. 1 and 3 are no longer valid. Also, when there is external noise, other memorized patterns may be excited destabilizing the initial configuration, where the system expressed one memorized information pattern.

We monitored several quantities. The first one is the overlap $\left\langle m^{\mu}\right\rangle_{t}$ of the network with the $\mu$ th pattern ( $\mu$ $=1,2, \ldots, P)$ at time $t$, defined as

$$
\left\langle m^{\mu}\right\rangle_{t}=\sum_{\sigma=0}^{2^{M}-1} \frac{y(\sigma, t)}{a(t)} m\left(\sigma^{\mu}, \sigma\right),
$$

where

$$
m\left(\sigma^{\mu}, \sigma\right)=\frac{1}{M} \sum_{i=1}^{M}\left[2 \delta\left(\sigma_{i}^{\mu}, \sigma_{i}\right)-1\right],
$$

with $\sigma_{i}^{\mu}$ and $\sigma_{i}$ being the $i$ th bits of, respectively, $\sigma^{\mu}$ and $\sigma$, and $\delta$ is the Kronecker delta function.

We also keep track of the information intensity of a given pattern $y_{0}^{\mu}(t)=y\left(\sigma^{\mu}, t\right)$ and of its neighborhood $y_{1}^{\mu}(t)$, defined as

$$
y_{1}^{\mu}(t)=\frac{1}{M} \sum_{i=1}^{M} y\left(\sigma^{i(\mu)}, t\right)
$$

where $\sigma^{i(\mu)}$ is the neighbor of $\sigma^{\mu}$ with the $i$ th bit flipped. We finally measure the information activity $a(t)$ is defined in Eq. (2), and the background $b^{\mu}(t)$ related to the $\mu$ th stored pattern as

$$
b^{\mu}(t)=a(t)-y_{0}^{\mu}(t)-M y_{1}^{\mu}(t)
$$




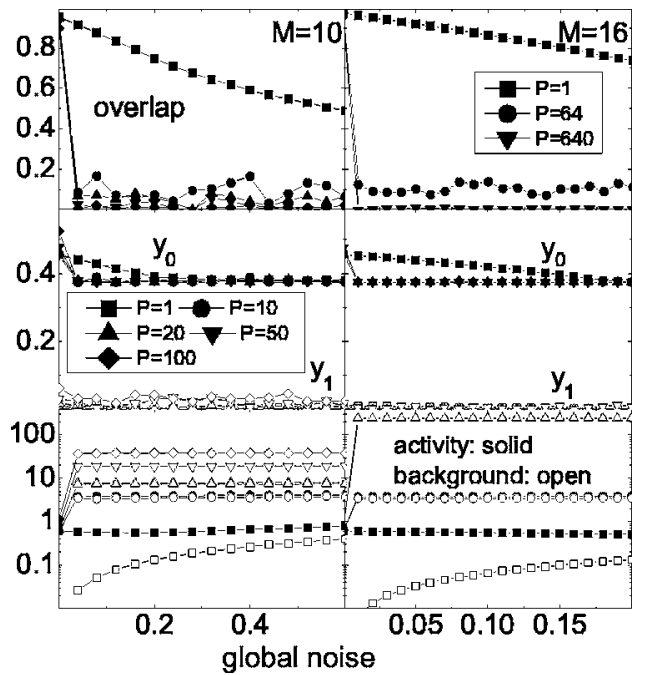

FIG. 4. Behavior of the model as function of global external noise for $k_{m}=1.6, k_{v}=0.25$, and $z=1.0$. Upper plots: Overlap with an initially excited memory. Middle plots: values of the intensity function $y_{0}$ for the excited pattern and the average $y_{1}$ for its neighborhood. Lower plots: global activity $a$ and background $b$ related to the initially excited pattern. Left column: $M=10$. Right column: $M=16$.

which gives the network excitation that is not caused by the excitation of $\sigma^{\mu}$

We have measured the above defined quantities after iterating Eq. (5) for long time, starting from the $M \rightarrow \infty$ stationary solutions given by Eq. (16), subjecting the system to noise and different number $P$ of memorized patterns. The noise is introduced by adding to all intensity functions a random term, uniformly distributed in the interval $\left[0, T / 2^{M}\right]$, after each time step iteration has been carried out. The factor $1 / 2^{M}$ allows us to compare different $M$ simulation runs with the same value of global noise $T$, since at each time step this is equivalent to add $T / 2$ to the global information activity $a(t)$. The results in Figs. 4-6 have been obtained after averaging over ten samples.

We considered different regions of the phase diagram. We start with the case where $k_{m}>1$ and $k_{v}+z>1$. In this case

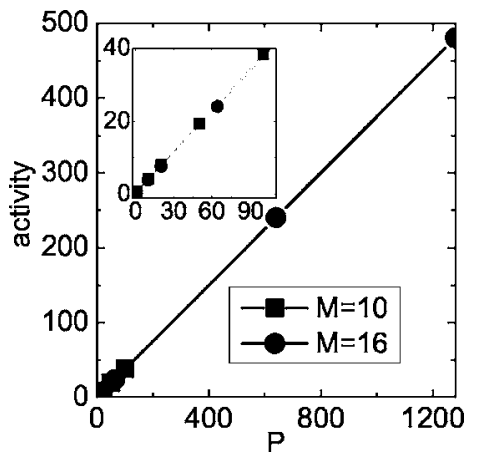

FIG. 5. Global activity $a$ as function of network load $P$ for $k_{m}$ $=1.6, k_{v}=0.25$, and $z=1.0$, in the presence of a nonzero external noise: $a$ goes linearly with $P$ indicating that all memorized patterns are excited by the external noise. We show results for $M=10$ and $M=16$. The inset shows the linear dependence for initial values of $P$.

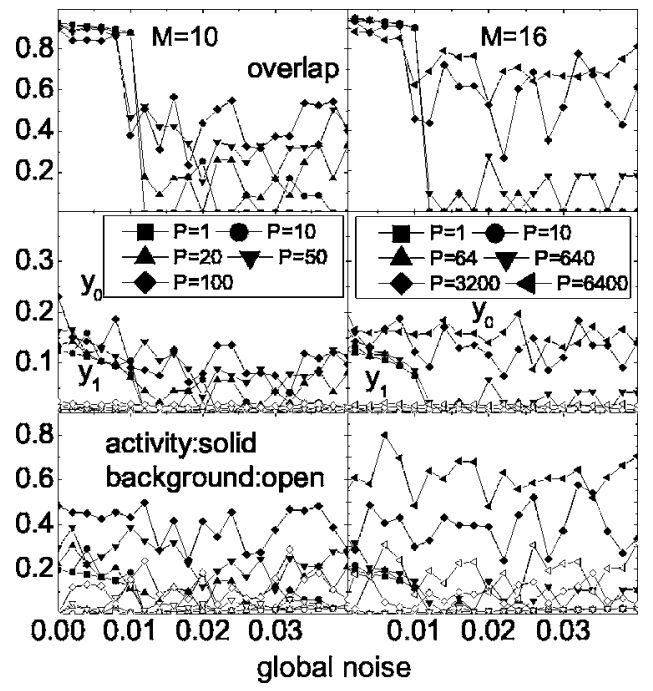

FIG. 6. Behavior of the model as function of global external noise for $k_{m}=0.7, k_{v}=0.25$, and $z=1.2$. Upper plots: Overlap with an initially excited memory. Middle plots: values of the intensity function $y_{0}$ for the excited pattern and the average $y_{1}$ for its neighborhood. Lower plots: global activity $a$ and background $b$ related to the initially excited pattern. Left column: $M=10$. Right column: $M=16$.

the one memory stationary solutions in the absence of noise is stable when $1<k_{m}<3$ and a memorized pattern may stay excited without the support of neighboring sites. This means the expression of memorized patterns are not strongly affected by the global activity of the network and we expect that noise awakes other memorized patterns.

In Fig. 4 we present the results for this case and we see that for all values of nonzero noise the overlap is very near zero, although the expression intensity $y_{0}$ of the initial pattern is kept at non-negligible values. It means that all other memorized patterns are also excited, yielding an activity that grows linearly with $P$, shown in Fig. 5. As a consequence, this is not an interesting region for modeling associative memory. We also report having considered the case $k_{m}>1$, but $k_{v}+z<1$. The results are not significantly different from the above case, when $k_{m}>1$, and $k_{v}+z>1$ : it suffices that $k_{m}>1$ for all stored patterns to excite in presence of noise.

We considered then the case when $k_{m}<1$, and $k_{v}+z>1$. This region is interesting because a memorized pattern needs the support of the neighboring sites in order to keep a finite intensity function. The results are shown in Fig. 6. We can observe a transition from regions where a finite value of overlap with the initially excited pattern is maintained, to another behavior where this overlap decreases to values near zero. The nature of this transition strongly depends on $P$ : for small values of $P$ the system suffers a first order transition while, as $P$ increases, the transition from high to low values of final overlap seems to be gradual. We also remark that for different network sizes, the values of $P$ where there is a deviation from the first order transition varies, scaling with $2^{M}$. This seems to be a percolation induced effect: as the network is loaded, the probability that excited stored patterns influences their neighbors increases and the stability of one retrieved memory is disturbed. Figure 6, together with the 


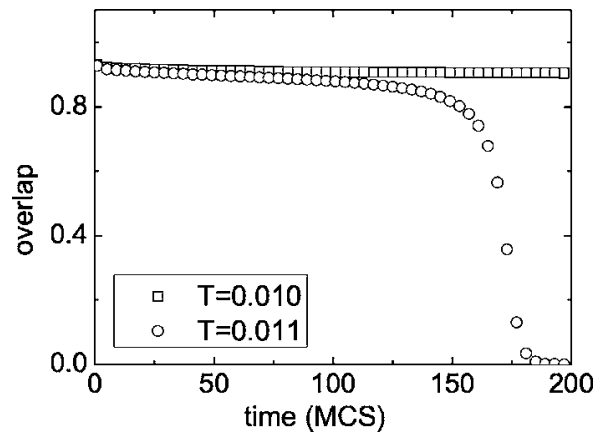

FIG. 7. Overlap with an initially excited memory for $M=16$, $P=120, k_{m}=0.7, k_{v}=0.25$, and $z=1.2$, considering two values of external noise $T=0.010$ and 0.011 , respectively, before and after the transition.

results from previous sections, indicate that the interesting regions in the phase space to model associative memory is the one defined by the constraints $k_{m}<1, k_{v} \leqslant k_{m}, 2<k_{m}$ $+k_{v}+z<4$, and $k_{v}+z>1$.

On the other hand, for high values of network load $P$ and noise $T$, the overlap fluctuates wildly, but with nonneglegible, positive values, while the network activity $a$ is significantly larger than the background $b$. It indicates that the initial pattern is still the main pattern expressed by the net. In that sense, loading the network helps maintaining an initially expressed pattern against external noise.

As far as noise is concerned, however, Fig. 6 does not tell the whole story. In Fig. 7 we plot the overlap with the initially excited pattern versus time for $P=120$ for $M=16$, for a noise lower $(T=0.010)$ and higher $(T=0.011)$ than the critical value. In accordance to Fig. 6, the overlap either keeps at a finite value or goes to zero, depending on the noise. However, for $T>T_{c}$, there is a finite time interval when the overlap is kept at high values: the network is capable of retrieving an excited pattern for a finite time when subjected to noise, after which it forgets. It would be interesting to measure the dependence of the retrieving time interval with the load $P$, and noise level, but it is beyond the scope of the present work.

\section{BASINS OF ATTRACTION}

We are finally at a stage where we can consider estimating basins of attraction. Basins of attraction measures are intended to estimate the maximum distance from a memorized pattern that an initial stimulus is able to make the system recover that pattern. We have considered different levels of external noise and systems with only one memorized patterns to avoid competing effects from nearby memories. The simulation protocol we used is as follows. We first stabilized the system by letting it relax for 20 time steps subject to the given external noise. A randomly chosen information pattern, in a $h_{0}$ Hamming distance from the memorized pattern is stimulated for 40 time steps, that is, after each time step we added a fixed amount to the stimulated pattern expression intensity. We then let the system evolve, always subject to the same external noise for 1000 time steps, when the system is already stabilized. We then measured the expression intensity of the memorized and stimulated pattern, as well as the global activity of the network for different values of $k_{m}<1$, $k_{v}$, and $z$.

In Fig. 8 we present the results for $M=16, k_{m}=0.7,0.8$, and 0.9 , with $k_{v}=0.0,0.05,0.15,0.25$, and 0.35 , and with the coupling constant $z=1.05,2.05$, and 3.05. Different levels of external global noise $T$ were also considered.

For stimuli with three or more bits different from the memorized pattern we could not find any memory recovery. The final configuration is either no excited pattern at all, or only the stimulated pattern excited. This last situation happens for $2 k_{v}+z>2$ which, in the theoretical solutions, corresponds to the recovery region for $k_{m}=k_{v}$. In Fig. 8 we have then presented the results for $h_{0}=0,1$, and 2. In that table we used a color code to represent the results for each set of parameters $k_{m}, k_{v}, z, h_{0}$ and $T$, such that each one of the four possible behaviors is associated to a color, as follows: (i) light gray means that both stimulated and memorized patterns are excited, (ii) shaded means that only the stimulated pattern is excited, (iii) dark gray only the memorized pattern is excited, and finally, (iv) white is used when no pattern is excited. In case $h_{0}=0$, that is the stimulus is applied on the memorized pattern, the awakening of the memorized pattern implies in the excitation of the stimulated pattern since they are the same, and the only possible colors are light gray or white.

For example, take $k_{m}=0.80$ and $T=0.001$. For $z=1.05$, $k_{v}=0.15$, the table in Fig. 8(b) shows white squares for $h_{0}$ $=0$, 1 , and 2, meaning that for stimuli at the memorized pattern, one or two bits apart no pattern is excited. For $z$ $=1.05$ and $k_{v}=0.35$ we find in the same table light gray, light gray, and white squares for, respectively, $h_{0}=0,1$, and 2 , indicating that applying the stimulus at the memorized pattern or at one of its neighbors, both memory and stimulated pattern stays excited while the stimulus at two bits apart cannot excite any pattern. Changing to $z=2.05$ and $k_{v}=0.15$, we find light gray, light gray and shaded squares for, respectively, $h_{0}=0,1$, and 2 , indicating that applying the stimulus at the memorized pattern or at one of its neighbors excites both the memorized pattern and the pattern at which the stimulus was applied, while applying the stimulus on a pattern that is two bits away excites only that pattern.

A different situation happens when $z=3.05$ and $k_{v}=0.15$ : the result is light, light, and dark gray squares for, respectively, $h_{0}=0,1$, and 2 . Now, when the stimulus is applied two bits from the memory, the memory is successfully retrieved with the stimulated pattern being activated only for a transient time.

For $z=3.05$ and $k_{v}=0.35$ the result is light gray, for $h_{0}$ $=0,1$ and 2 . Therefore both memorized and stimulated patterns are active for stimulations up to 2 bits away from the memory. This is an indication that $k_{v}$ and $z$ are high enough to cause a $y_{2} \neq 0$ stable state.

Four of these situations are further illustrated in Fig. 9. There we present the expression intensities for the memory $y_{\text {mem }}$ and the stimulated pattern $y_{\text {stim }}$ for stimuli at the memory, one or two bits apart. The two lower plots of Fig. 9 illustrate cases where $y_{0}$ is not stationary, but oscillates as it could be expected for $k_{m}+k_{v}+z \geqslant 4.00$. 


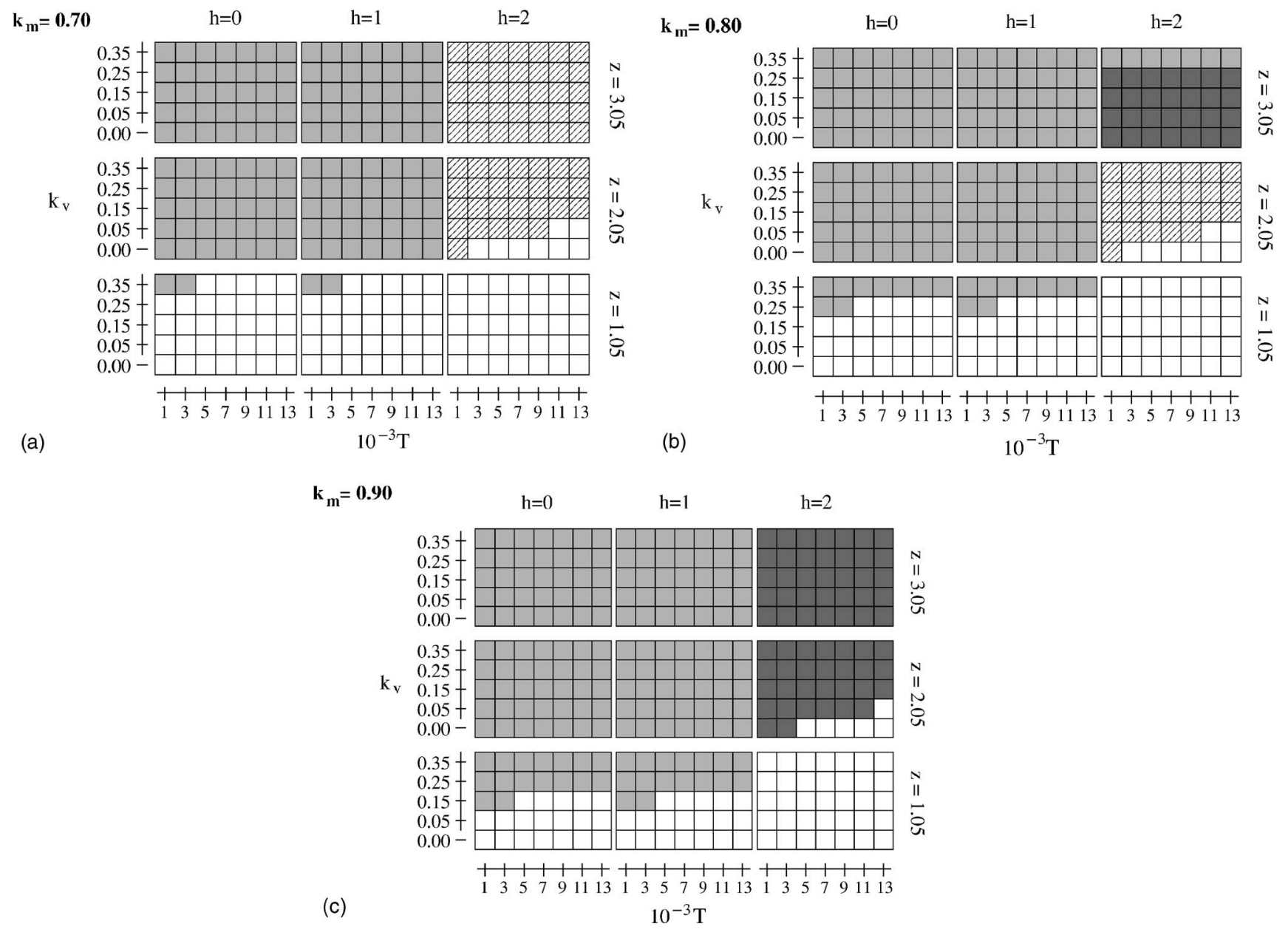

FIG. 8. Tables summarizing the pattern recovery from stimuli applied at Hamming distance $h_{0}=0,1,2$ from a memorized pattern. $M$ $=16$ and $P=1$. Light gray: both the memory and the stimulated patterns are excited. Shaded: only the stimulated pattern is excited. Dark gray: only the memorized pattern is excited. White: no pattern is excited.

Analyzing Figs. 8 and 9 the role of each parameter is uncovered. In summary, for an excitation to be transported from a stimulated pattern towards a not neighboring memory, it is necessary that the site in the middle is susceptible enough to be excited by a neighboring stimulus, but unstable enough to decrease its expression intensity after the memorized pattern is awaken. So $k_{v}$ cannot be too low to account for the intermediate state susceptibility, and noise may help at destabilizing it after the memorized pattern is excited. Furthermore, higher values of $k_{m}$ favor memory expression in respect to intermediate states, destabilizing further excited nonmemory states. This happens due to memory higher intensity expression that increases the whole network activity $a$ and decreases the effective logistic parameter $\lambda$ of nonmemories.

\section{EFFECTIVE BASINS OF ATTRACTION AND INFORMATION TRANSPORT}

We now consider the effect of loading the network on information transport and the related size of basins of attractions. By information transport we understand the excitation

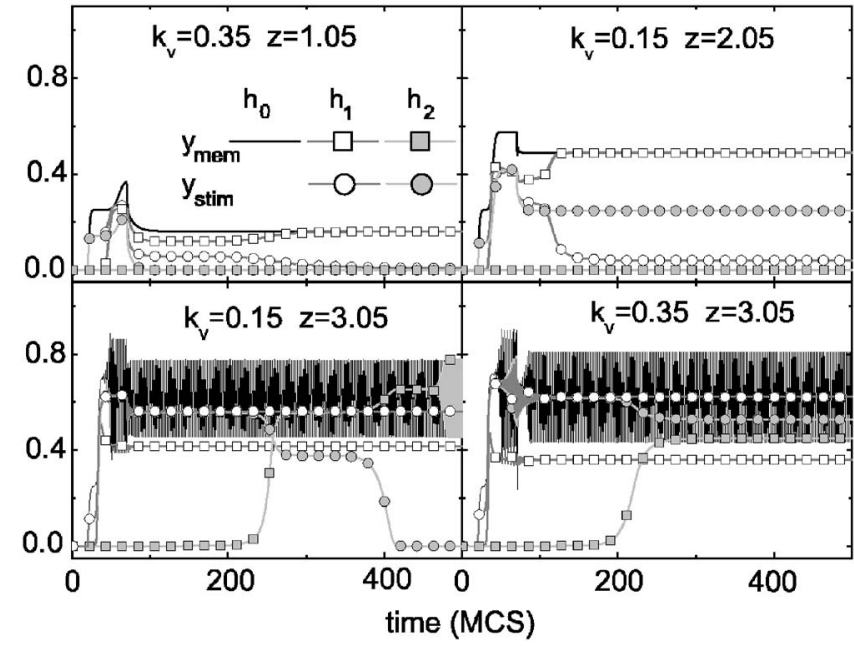

FIG. 9. Evolution of the expression intensities for the memory $y_{\text {mem }}$ and the stimulated pattern $y_{\text {stim }}$ for stimuli at the memory $(h$ $=0)$, one $(h=1)$, or two $(h=2)$ bits apart. $M=16, k_{m}=0.8$, and $T$ $=0.001$. Other parameters as indicated in the figures. 


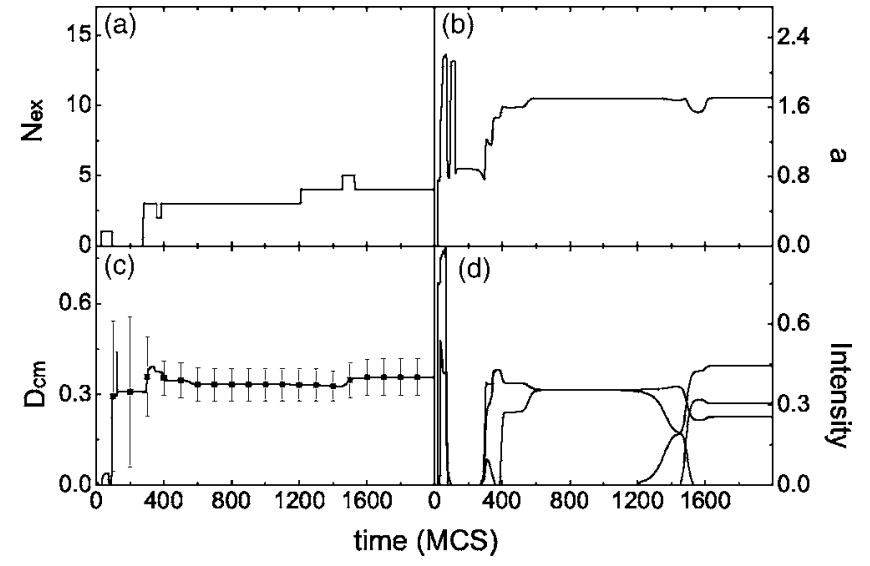

FIG. 10. Time evolution of (a) number of excited memories $N_{\text {ex }}$, (b) global activity $a$, (c) distance $D_{\text {c.m. }}$. of the excitation center of mass from the stimulated pattern, and (d) intensity of expression of excited memories. $M=16, P=3000$, and $T=0.001$.

of neighboring sites such that a cluster of awaken patterns either grow or moves in the information space. In the previous sections we investigated information transport in the case where the memories are not near each other. In this section we briefly discuss the opposite case: when $P$ is large enough such that stored memories are not isolated anymore on the information space.

In this case, the interaction between neighboring memories originates a complex behavior where a stimulus applied on a randomly chosen pattern may successively awaken a chain of memories until an interacting cluster of stored patterns stabilize at excited levels. To characterize and measure such a process, we followed the intensity of expression of all memories, the evolution of the network activity $a$, the number of excited memories $N_{\text {ex }}$, here defined as the number of memories being expressed with intensity $y>0.001$, and the distance $D_{\text {c.m. }}$ from the "excitation center of mass" position to the stimulated pattern $\sigma_{0}$, calculated as

$$
D_{\text {c.m. }}=\frac{1}{M} \frac{\sum_{\sigma=0}^{2^{M}-1} y(\sigma, t) h\left(\sigma, \sigma_{0}\right)}{\sum_{\sigma=0}^{2^{M}-1} y(\sigma, t)},
$$

where $h\left(\sigma, \sigma_{0}\right)$ is the number of different bits between $\sigma$ and $\sigma_{0}$, that is, the Hamming distance.

We considered $k_{m}=0.8, k_{v}=0.25, z=2.05$, being in the region where for $P=1$ and $h_{0}=2$ a single memory is retrieved, for $M=16$ systems. In Fig. 10 we show the results for $P$ $=3000$ and $T=0.001$. We have relaxed the network for 20 MCS at $T=0.001$ and have then randomly chosen a pattern. An external stimulus has been applied to this pattern by repeatedly summing 0.1 to its intensity of expression for 50 MCS. In Fig. 10 we show $a, N_{\text {ex }}$, and $D_{\text {c.m. }}$, together with the intensity of expression of the awaken memories and of the stimulated pattern. For very early time Fig. 10(d) presents two narrow peaks, one for the stimulated pattern and a second one for an awaken memory, in agreement with Fig. 10(a)

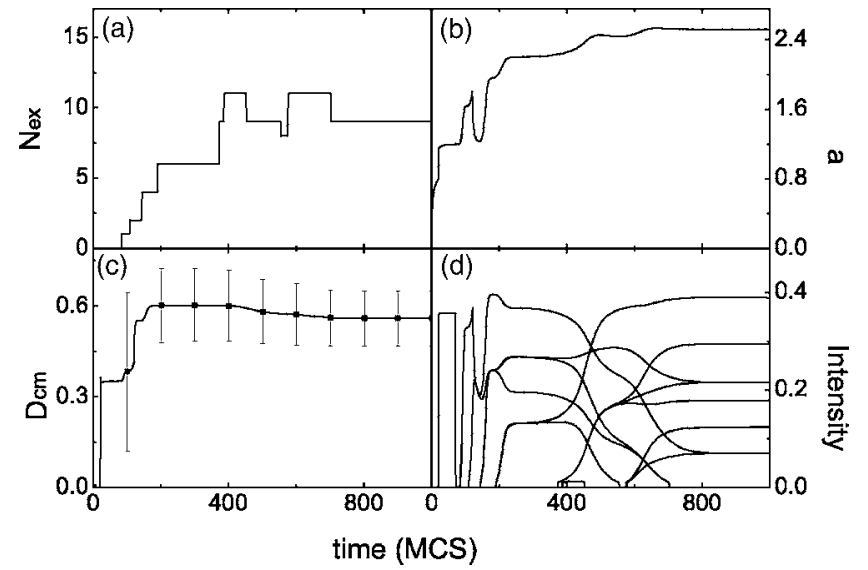

FIG. 11. Time evolution of (a) number of excited memories $N_{\text {ex }}$, (b) global activity $a$, (c) distance $D_{\text {c.m. }}$ of the excitation center of mass from the stimulated pattern, and (d) intensity of expression of excited memories. $M=16, P=6000$, and $T=0.8$.

showing $N_{\mathrm{ex}}=1$. This happens near the stimulated pattern as indicated by low values of $D_{\text {c.m. }}$. It follows then an interval, for $t<400 \mathrm{MCS}$, where no memory is being expressed, but the activity of the network does not go to zero, indicating that nonmemorized patterns are being expressed: neighboring patterns are being contaminated. For $t>400$, three memories are awaken and $D_{\text {c.m. }}$ increases, together with the network activity. For $400<t<1200$ the network seems stabilized, expressing four memories at $D_{\text {c.m. }}=0.345$. At roughly $t=1200 \mathrm{MCS}$, however, all four memories are forgotten, awakening other five, and, although the plots do not show that far, we report that the system stabilizes at this attractor as far as $t=10000 \mathrm{MCS}$.

We also show the results for $T=0.8$ and $P=6000$, in Fig. 11. The number of memories in the awakened clusters is higher and so are the network activity as well as $D_{\text {c.m. }}$, but qualitatively the same scenario appears: the external stimulus has caused the retrieval of a correlated cluster of memorized patterns by passing through a nontrivial transient when other memories have been expressed for reasonably long time intervals.

The characteristics of the attractors depend on $P$ and $T$. For very small values of $P$ there is not such transient and typically only stimulus at one or two bits apart a memory are able to retrieve it, as discussed for $P=1$ in the previous sections. For high values of $T$ it may happen that no memory is permanently awaken. The number of memories in the attractor also depends on $P$ and $T$.

On the other hand, the behavior we just described may be interpreted as the retrieval of very far memories, indicating an effective size of basins of attraction much larger than 2 bits. It is true also that, in this case, the attractors are not isolated memories any more.

\section{DISCUSSION AND CONCLUSION}

We analyzed a model for information processing machines, obtaining analytical and simulation results in order to characterize the model behavior. We started by discussing the 
possibility of going from the configuration space of a neural network to the information space. We then proposed novel equations to model the evolution of the expression intensity functions associated to every information pattern. An important advantage of the new model is its capability of simultaneously expressing many different information patterns.

It is worth mentioning that Treves and collaborators $[38,39]$ have proposed a Potts model with the interesting behavior where a sequence of attractors dynamically replace each other, also generating a concatenated retrieval of memorized patterns. However, as the dynamics is implemented in the configuration space, it is not possible to retrieve more than one information pattern at a given time. In fact, whenever the information space is implicitly taken as the configuration space, the simultaneous retrieval of many information patterns is not possible.

We then turned to analyze the model by describing the possible retrieval solutions in the limit of isolated memories. The results are acceptable but are not impressive. However, when the number of memorized patterns is enhanced, the scenario changes dramatically.

First, there is a qualitative change in the attractors of the network dynamics; from single, isolated patterns the attractors turn into clusters of stored information patterns. This characteristic is very interesting in the sense it can describe natural memory retrieving process, where, for example, different information association is triggered by a single information stimulation. Second, there is the very retrieving process, that first awakens transient memory patterns to then stabilize at the attractor cluster, perhaps emulating human remembering process where a chain of correlated information is successively recalled to eventually recover a given memory.
Regarding the details of the particular dynamics chosen here, it interesting to notice that $P=6000$ in Fig. 11 is beyond the limit of bootstrap percolation [37], meaning that all the existent memory clusters are connected by jumps of 2 sites. The same is probably true for $P=3000$ in Fig. 10 as well, since Ref. [37] only presents lower and upper limits for the critical probability.

Therefore, our dynamics for $y_{1} \neq 0$ sees only a single percolating cluster and it is surprising that so few memories are awaken after all. A possible explanation comes from the role of $a(t)$ in limiting excitation. When a memory is stimulated it causes a spread of activity, such as a chain reaction in an inhomogeneous media. But as the wave of activity grows it gets weak and incapable of further propagation. Then the center of mass drifts towards a region with larger memory density. This might indicate that there are many possible stable clusters of activity depending on the initial stimulus.

The previous section left open many questions. It presented a model with a novel behavior, which requires the careful definition of order parameters and relevant quantities that will allow its adequate description. For example, one should estimate the number of stable attractors and the size of the effective basins of attraction, how they depend on temperature, and also design different stimulation protocols to compare the model behavior with known human performances in realizing tasks, such as recalling items from a previously studied list. These points are presently under investigation and the results will be presented elsewhere.

\section{ACKNOWLEDGMENTS}

We acknowledge partial financial support from Brazilian agencies FAPERGS, CNPq, and CAPES.
[1] R. M. C. de Almeida and M. A. P. Idiart, Phys. Rev. E 65, 061908 (2002).

[2] J. Herz, A. Krogh, and R. G. Palmer, Introduction to the Theory of Neural Computation (Addison-Wesley, New York, 1991).

[3] H. S. Seung, H. Sompolinsky, and N. Tishby, Phys. Rev. A 45, 6056 (1992).

[4] D. J. Amit, Modeling Brain Function: The World of Attractor Neural Networks (Cambridge University Press, New York, 1989).

[5] T. L. H. Watkin, A. Rau, and M. Biehl, Rev. Mod. Phys. 65, 499 (1993).

[6] C. M. Gray, W. Singer, Proc. Natl. Acad. Sci. U.S.A. 86, 1698 (1989)

[7] E. Vaadia, I Haalman, M. Abeles, H. Bergman, Y. Prut, H. Slovin, and A. Aertsen, Nature (London) 373, 515 (1995).

[8] W. Singer, Neuron 24, 49 (1999).

[9] P. N. Steinmetz, A. Roy, P. J. Fitzgerald, S. S. Hsiao, K. O. Johnson, and E. Niebur, Nature (London) 404, 187 (2000).

[10] Z. Nádasdy, J. Physiol. (Paris) 94, 505 (2000).

[11] M. Galarreta and S. Hestrin, Science 292, 2295 (2001).

[12] S. Hestrin and M. Galarreta, Trends Neurosci. 28, 304 (2005).
[13] V. B. Mountcastle, in The Mindful Brain, edited by G. M. Edelman and V. B. Mountcastle (MIT Press, Cambridge, MA, 1978), p. 1.

[14] V. B. Mountcastle, Brain 120, 701 (1997).

[15] V. B. Mountcastle, Perceptual Neuroscience: The Cerebral Cortex (Harvard University Press, Cambridge, MA, 1998).

[16] D. Hubel and T. Wiesel, J. Physiol. (London) 160, 106 (1962).

[17] D. Hubel and T. Wiesel, J. Physiol. (London) 195, 215 (1968).

[18] P. S. Goldman-Rakic, Trends Neurosci. 7, 425 (1984).

[19] P. Rakic, Science 241, 170 (1988).

[20] G. L. Shaw, D. J. Silverman, and D. J. Pearson, Proc. Natl. Acad. Sci. U.S.A. 82, 2364 (1985).

[21] M. Sardesai, C. Figge, M. Bodner, M. Crosby, J. Hansen, J. A. Quillfeldt, S. Landau, A. Ostling, S. Vuong, and G. L. Shaw, Biol. Cybern. 84, 173 (2001).

[22] A. Damásio, Descartes' Error: Emotion, Reason, and the Human Brain (Grosset/Putnam, New York, 1996).

[23] A. Damásio, The Feeling of What Happens (Harcourt, Brace, New York, 1999).

[24] J. LeDoux, The Emotional Brain (Simon and Schuster, New York, 1996).

[25] J.-D. Vincent, Biologie des Passions (Éditions Odile Jacob, 
Paris, 1999).

[26] G. M. Edelmann and G. Tononi, A Universe of Consciousness: How Matter Becomes Imagination (Perseus Books Group, New York, 2000).

[27] R. M. C. de Almeida and J. R. Iglesias, Phys. Lett. A 146, 239 (1990).

[28] J. J. Arenzon, R. M. C. de Almeida, and J. R. Iglesias, J. Stat. Phys. 69, 385 (1992).

[29] J. J. Arenzon and R. M. C. de Almeida, Phys. Rev. E 48, 4060 (1993).

[30] R. M. C. de Almeida, P. M. C. de Oliveira, and T. J. P. Penna, Annu. Rev. Comput. Phys. 1, 193 (1994).

[31] D. Bollé, J. Huyghebaert, and G. M. Shim, J. Phys. A 27, 5871
(1994).

[32] E. Botelho, R. M. C. de Almeida, and J. R Iglesias, J. Phys. A 28, 1879 (1995).

[33] R. M. C. de Almeida e E. Botelho, Physica A 242, 27 (1997).

[34] N. Cowan, Behav. Brain Sci. 24, 87 (2000).

[35] A. Baddeley, Human Memory: Theory and Practice, Revised Ed. (Allyn and Bacon, Boston, 1998).

[36] L. Postman and L. W. Phillips, Q. J. Exp. Psychol. 17, 132 (1965).

[37] J. Balogh and B. Bollobas, Probab. Theory Relat. Fields 134, 624 (2006).

[38] A. Treves, Neuropsychologia 22, 276 (2005).

[39] E. Kropff and A. Treves, J. Stat. Mech.: Theory Exp. (2005). 